\title{
Near-Field Diffraction from a Binary Microaxicon
}

\author{
Victor V. Kotlyar, ${ }^{1,2}$ Sergey S. Stafeev, ${ }^{1,2}$ Roman V. Skidanov, ${ }^{1,2}$ and Victor A. Soifer ${ }^{1,2}$ \\ ${ }^{1}$ Laser Measurements Laboratory, Image Processing Systems Institute, The Russian Academy of Sciences, \\ 151 Molodogvardeyskaya Street, Samara 443001, Russia \\ ${ }^{2}$ Technical Cybernetics Subdepartment, S. P. Korolyov Samara State Aerospace University, 34 Moskovskoe Shosse, \\ Samara 443086, Russia
}

Correspondence should be addressed to Sergey S. Stafeev, sergey.stafeev@gmail.com

Received 5 April 2012; Accepted 7 June 2012

Academic Editor: Maria Calvo

Copyright ( 2012 Victor V. Kotlyar et al. This is an open access article distributed under the Creative Commons Attribution License, which permits unrestricted use, distribution, and reproduction in any medium, provided the original work is properly cited.

\begin{abstract}
We study binary axicons of period 4,6, and $8 \mu \mathrm{m}$ fabricated by photolithography with a $1 \mu \mathrm{m}$ resolution, $500 \mathrm{~nm}$ depth, and $4 \mathrm{~mm}$ diameter. Near-field diffraction focal spots varying in diameter from $3.5 \lambda$ to $4.5 \lambda$ (for the axicon of period $T=4 \mu \mathrm{m}$ ) and from $5 \lambda$ to $8 \lambda$ (for the axicon with $T=8 \mu \mathrm{m}$ ) are experimentally found on the optical axis at a distance of up to $40 \mu \mathrm{m}$ from the axicon for the wavelength $\lambda=0.532 \mu \mathrm{m}$. The first focal spot is found at distance $2 \mu \mathrm{m}(T=4 \mu \mathrm{m})$, with the period of the focal spots being $2 \mu \mathrm{m}(T=4 \mu \mathrm{m})$ and $4 \mu \mathrm{m}(T=8 \mu \mathrm{m})$. Diffraction of linearly polarized plane and diverging waves is simulated using FullWAVE (RSoft) and a proprietary program BOR-FDTD, which implement finite-difference schemes to solve three-dimensional Maxwell's equations in the Cartesian and cylindrical coordinates. The numerically simulated values for diameters of the near-field focal spots for the axicon of period $T=4 \mu \mathrm{m}$ are in good agreement with the experimental values.
\end{abstract}

\section{Introduction}

Axicons [1] are known to be suitable for generating a diffraction-free laser Bessel beam in a definite range of the optical axis $[2,3]$. Such beams continue to attract researchers' interest. In [4] a coreless silica fiber of diameter $30 \mu \mathrm{m}$ and thickness $3 \mu \mathrm{m}$, combined with a lens of radius $70 \mu \mathrm{m}$ was used for generating a Bessel beam of diameter $20 \mu \mathrm{m}$ maintained over $500 \mu \mathrm{m}$ distance at wavelength $\lambda=$ $1.55 \mu \mathrm{m}$. In [5] the FDTD-method was used to model a $2 \mathrm{D}$ photonic crystal composed of an axicon-shaped rectangular array of dielectric rods: axicon base, $20 a$; height, $10 a$; refractive index of the rods, $n=3.13$; radius of the rods, $0.22 a$; wavelength, $\lambda=a / 0.36$, where $a$ is the period of the rod array. A diverging Bessel beam of diameter FWHM = $1.5 \lambda$ at half-maximum intensity was shown to be generated at a distance of $z<30 a$. A surface plasmon wave in the form of concentric rings described by the first-order Bessel function was reported in [6] in experiments using a radially polarized laser beam $(\lambda=532 \mathrm{~nm})$, a conical axicon, and an immersion microlens with numerical aperture $\mathrm{NA}=1.25$ found in a silver film of thickness $50 \mathrm{~nm}$ (permittivity $\varepsilon=$ $-10.1786-i 0.8238)$. The central axial ring diameter was $278 \mathrm{~nm}$ and the thickness was $250 \mathrm{~nm} \approx 0.5 \lambda$. The surface plasmon pattern was observed with a near-field microscope Veeco Aurora 3 with a $50-100 \mathrm{~nm}$ resolution. In a similar work [7], a scheme including a radially polarized beam of a He-Ne laser $(\lambda=632.8 \mathrm{~nm})$, an axicon, and an immersion lens with NA $=1.4$ in a $44 \mathrm{~nm}$ thick Au film $(\varepsilon=0.3+$ i3.089) was used to form a surface plasmon with a central focal spot of diameter FWHM $=0.22 \mu \mathrm{m}=0.35 \lambda$. The plasmon was observed with the aid of a latex ball $175 \mathrm{~nm}$ in diameter.

Focusing the laser light in the neighborhood of an annular structure on metal was discussed in $[8,9]$. In [8], the focusing of light with a zone plate of the ring radii $r_{n}{ }^{2}=2 n f \lambda+n^{2} \lambda^{2}, f=1 \mu \mathrm{m}, \lambda=633 \mathrm{~nm}$, was simulated by the FDTD method. The plate was realized in thin silver $(50 \mathrm{~nm})$ and golden $(50 \mathrm{~nm})$ films deposited on silica. The diameter of the ring array was $13 \mu \mathrm{m}$. A focal spot of halfintensity diameter FWHM $=0.3 \lambda$ (spot's full width being $0.7 \lambda$ ) was shown to occur at a distance of $z=1.5 \mu \mathrm{m}$ 
from the plate. Similar ring arrays $(8 \mu \mathrm{m}$ in diameter $)$ in a $100 \mathrm{~nm}$ thick golden film were experimentally obtained in [9]. Using a near-field microscope NTEGRA (NT-MDT) with $100 \mathrm{~nm}$ resolution, a focal spot of diameter FWHM = $0.5 \mu \mathrm{m}$ at half intensity (full diameter $5 \lambda$ ) was observed at distance $z=1.6 \mu \mathrm{m}$ for $\lambda=633 \mathrm{~nm}$. Note that the theoretical estimation for the focal spot gives FWHM = $0.5 \lambda$. In [10], eight nanoholes of diameter $200 \mathrm{~nm}$ arranged symmetrically in a circle of diameter $1 \mu \mathrm{m}$ in the PMMA resist on glass were illuminated with the incoherent light of wavelength $\lambda=650 \mathrm{~nm}$, producing a focal spot of size $\mathrm{FWHM}=0.4 \lambda$ (full diameter $1.2 \lambda$ ) at distance $500 \mathrm{~nm}$ from the surface. A Fresnel lens of focal length $f=5 \mu \mathrm{m}$ and diameter $50 \mu \mathrm{m}$ in the $120 \mathrm{~nm}$ thick amorphous silicon film was realized in [11] for wavelength $\lambda=575 \mathrm{~nm}$, offering a $26 \%$ transmittance. Upon immersion, the lens numerical aperture was NA $=1.55$, producing a focal spot of diameter FWHM $=0.9 \lambda$. It is noteworthy that the spot was measured using a fluorescent sphere of diameter $0.5 \mu \mathrm{m}$.

Other theoretical [12] and experimental [13] works reported on the studies into the near-field focusing of light with a binary diffraction axicon. An approximate theory that adequately described a diffraction axicon of ring period $T<5 \lambda$ was proposed in [12]. In the said theory, with its central part shielded with an opaque disk, the binary axicon can be treated as a diffraction grating. The axicon of period $T=5 \lambda$ and radius $40 \lambda$ was shown to produce a focal spot of diameter FWHM $=0.88 \lambda$ at distance $40 \lambda$ from the surface. Experimental studies of a binary axicon $(30 \mathrm{~mm}$ in diameter) of period $T=33 \mu \mathrm{m}$ (which is equivalent to a conical glass axicon with the apex angle of $88^{\circ}$ ) fabricated on the ZEP520A resist (refractive index $n=1.46$ ) was reported in [13]. The laser beam diameter was shown to be independent of the wavelength, with the Bessel beam radius increasing from $1.2 \mu \mathrm{m}$ to $12.5 \mu \mathrm{m}(\lambda=532 \mathrm{~nm})$ over a distance range from 0 to $z=50 \mathrm{~mm}$, retaining its radius upon further propagation from $z=50 \mathrm{~mm}$ to $z=$ $100 \mathrm{~mm}$.

In this work, we study binary axicons of period 4,6 , and $8 \mu \mathrm{m}$ fabricated by photolithography with a $1 \mu \mathrm{m}$ resolution, of depth $500 \mathrm{~nm}$, and diameter $4 \mathrm{~mm}$. We experimentally show that the near-field diffraction produces focal spots within a $40 \mu \mathrm{m}$ distance from the axicon, varying in diameter from $3.5 \lambda$ to $4.5 \lambda$ (for the axicon with $T=4 \mu \mathrm{m}$ ) and from $5 \lambda$ to $8 \lambda$ (for $T=8 \mu \mathrm{m}$ ), $\lambda$ is the incident wavelength $(\lambda=0.532 \mu \mathrm{m})$. Note that with the first focus appearing at distance $2 \mu \mathrm{m}(T=4 \mu \mathrm{m})$, the focal spots recur with a period of $2 \mu \mathrm{m}$ (for $T=4 \mu \mathrm{m}$ ) and $4 \mu \mathrm{m}$ (for $T=8 \mu \mathrm{m}$ ). We have also modeled the diffraction of plane and diverging linearly polarized light waves using FullWAVE (RSoft) and a proprietary program BOR-FDTD that use finite-different schemes to solve Maxwell's equations in the Cartesian and cylindrical coordinates. The numerical values of paraxial focal spot diameters for the near-field diffraction derived for the axicon with period $T=4 \mu \mathrm{m}$ are in good agreement with the experimental results.

\section{Scalar Nonparaxial Diffraction by a Binary Axicon}

The approximate theory [12] is not suited for the analysis of the near-axicon field, because the central part of the axicon cannot be treated as a diffraction grating.

With a plane linearly polarized wave incident on the axicon, the electric field of the binary axicon in the initial plane $z=0$, which is coincident with the axicon output surface, is given in the transparency approximation by

$$
E_{y 0}(r)=\left(1-e^{i \phi}\right) \sum_{n=0}^{N-1}(-1)^{n} \operatorname{circl}\left(\frac{r}{r_{n}}\right),
$$

where $r_{n}=(n+1) r_{0}$, nis integer, $r_{n}$ are radii of the axicon binary relief steps along the radial coordinate $r, \varphi$ is the phase delay due to the axicon relief steps, and $N$ is the number of jumps of the axicon relief. The amplitudes of the spectrum of plane waves given by the initial field in (1) are derived from the expression

$$
\begin{aligned}
A(\rho) & =\frac{k^{2}}{2 \pi}\left(1-e^{i \phi}\right) \sum_{n=0}^{N-1}(-1)^{n} \int_{0}^{\infty} \operatorname{cicrl}\left(\frac{r}{r_{n}}\right) J_{0}(k r \rho) r d r \\
& =\left(1-e^{i \phi}\right) k^{2} r_{0}^{2} \sum_{n=0}^{N-1}(-1)^{n}(n+1)^{2} \frac{J_{1}\left[k \rho(n+1) r_{0}\right]}{\left[k \rho(n+1) r_{0}\right]}
\end{aligned}
$$

where $\rho$ is a dimensionless variable.

Then, the $y$-component of the electric field in any plane $z$ is derived from

$$
\begin{aligned}
& E_{y}(r, z)=\left(1-e^{i \phi}\right) k^{2} r_{0}^{2} \sum_{n=0}^{N-1}(-1)^{n}(n+1)^{2} \\
& \int_{0}^{\infty} \frac{J_{1}\left[k r_{0}(n+1) \rho\right]}{\left[k r_{0}(n+1) \rho\right]} J_{0}(k \rho r) e^{i k z \sqrt{1-\rho^{2}}} \rho d \rho .
\end{aligned}
$$

The problem of finding the light field amplitude in the neighbourhood of the binary axicon (3) reduces to taking the integral:

$$
I=\int_{0}^{\infty} J_{1}(\alpha x) J_{0}(\beta x) e^{i \gamma \sqrt{1-x^{2}}} d x
$$

where $\alpha, \beta$, and $\gamma$ are constant numbers. Since we have failed to find the integral (4) in the reference literature, we will calculate it in the limiting cases. Assuming $\gamma=k z \ll 1$, we will find the near-axicon field. Then, by expanding the exponent into the Taylor series and retaining two terms, we obtain, instead of (4),

$$
\begin{aligned}
I \approx & \int_{0}^{\infty} J_{1}(\alpha x) J_{0}(\beta x) d x \\
& +i \gamma \int_{0}^{\infty} \sqrt{1-x^{2}} J_{1}(\alpha x) J_{0}(\beta x) d x .
\end{aligned}
$$

The first integral can be found in a reference book [14]:

$$
\int_{0}^{\infty} J_{1}(\alpha x) J_{0}(\beta x) d x= \begin{cases}\frac{1}{\alpha}, & \alpha>\beta, \\ 0, & \alpha<\beta .\end{cases}
$$


Note that in our case $\alpha=k r_{0}(n+1), \beta=k r$. The second integral in (5) will be sought for near the optical axis, on the assumption that the radial coordinate $r$ is much smaller than the radius of the axicon relief first step $r_{0}: r \ll r_{0}$; then the zero-order Bessel function can be replaced with the quadratic relationship: $J_{0}(x) \approx 1-(x / 2)^{2}$ at $x \ll 1$. This is done; the second integral in (5) can be derived using the reference integral [14]:

$$
\begin{aligned}
\int_{0}^{1} x^{a-1}\left(1-x^{2}\right)^{b-1} J_{n}(c x) d x \\
=\frac{c^{n}}{2^{n+1}} \frac{\Gamma(b) \Gamma((n+a) / 2)}{\Gamma(b+(n+a) / 2) \Gamma(n+1)} \\
\quad \times{ }_{1} F_{2}\left[\frac{n+a}{2}, \frac{n+a}{2}+b, n+1,-\frac{c^{2}}{4}\right],
\end{aligned}
$$

where ${ }_{1} F_{2}(a, b, c, x)$ is a hypergeometric function. Then, for the second integral in (5) we can write down the expression:

$$
\begin{aligned}
& \int_{0}^{1} \sqrt{1-x^{2}} J_{1}(\alpha x) d x-\left(\frac{k r}{2}\right)^{2} \int_{0}^{1} x^{2} \sqrt{1-x^{2}} J_{1}(\alpha x) d x \\
& =\left(\frac{k r_{0}}{6}\right)^{2}\left\{{ }_{1} F_{2}\left(1, \frac{5}{2}, 1,-y^{2}\right)-\frac{(k r)^{2}}{10}{ }_{1} F_{2}\left(2, \frac{7}{2}, 2,-y^{2}\right)\right\},
\end{aligned}
$$

where $y=k r_{0} / 2$. Replacing the infinite limits of integration in (5) by finite limits of integration in (8) is a routine procedure in which the contribution of evanescent inhomogeneous waves into the amplitude is disregarded.

In view of (6) and (8), the paraxial and near-axicon field $\left(k z \ll 1, r \ll r_{0}\right)$ is given by

$$
\begin{aligned}
& E_{\mathrm{y}}(r, z) \\
& \qquad \frac{1}{k r_{0}}+i k z \frac{\left(k r_{0}\right)}{6} \\
& \quad \times\left[{ }_{1} F_{2}\left(1, \frac{5}{2}, 2,-y^{2}\right)-\frac{(k r)^{2}}{10}{ }_{1} F_{2}\left(2, \frac{7}{2}, 2,-y^{2}\right)\right] .
\end{aligned}
$$

From (9), the diameter of the central peak of the paraxial light field is given by the relation

$$
2 r=k^{-1}\left[\frac{10_{1} F_{2}\left(1, \frac{5}{2}, 2,-y^{2}\right)}{{ }_{1} F_{2}\left(2, \frac{7}{2}, 2,-y^{2}\right)}\right]^{1 / 2},
$$

whence follows the numerical estimate of the focus diameter:

$$
2 r \approx 0.6 \lambda .
$$

The relation in (11) suggests that the central peak diameter of the near-axicon field is independent of its period $T=2 r_{0}$, being nearly equal to the diffraction limit (FWHM $=0.51 \lambda$ ).

To verify the expression (11), we can evaluate the central peak diameter of the field intensity based on different considerations.
2.1. Paraxial Estimate of the Axial Beam Diameter. In [13] the scalar paraxial theory was employed to show that since the axicon generates a Bessel beam, the Bessel beam diameter can be deduced from the expression:

$$
J_{0}^{2}(k \sin \theta \cdot r)=0 .
$$

Then, we obtain

$$
2 r=\frac{2.4 \lambda}{\pi \sin \theta},
$$

where $\theta$ is half angle at the apex of a conical wave generated by the axicon.

With the binary axicon treated as a diffraction grating [12], the conical wave angle $\theta$ can simultaneously be interpreted as the angle of a diffraction grating with period $T$ :

$$
\sin \theta_{m}=\frac{\lambda m}{T},
$$

where $m$ is the diffraction order number.

In view of (13) and (14), the final estimate of the axicon field diameter on the optical axis is

$$
2 r=\frac{2.4}{\pi} \frac{T}{m} \approx 0.774 \frac{T}{m} .
$$

From the relation (15) it can be seen that the Bessel beam diameter produced by the binary axicon is independent of the wavelength [13], only depending on the axicon period and the diffraction order number. The relation (15) suggests that different diffraction orders reach the near-axicon axial point and thus the light field formed in the axicon vicinity at $z<z_{0}$ (where $z_{0}=R T / 2 \lambda$ is the distance beyond which a single diffraction order of the axicon field is produced and $R$ is the axicon radius) will have the diameter varying in a complex manner along the optical axis at $0<z<z_{0}$.

From (15) it follows that at $z \geq z_{0}$ the axial beam diameter is equal to $2 r=6.2 \lambda$ for the axicon with period $T=4 \mu \mathrm{m}=8 \lambda$.

2.2. Estimating the Diameter of the Axial Beam as a Waveguide Mode. In the axicon vicinity, when $r<r_{0}=T / 2$ and $z<T$, we can estimate the axial light field diameter using the waveguide theory, because the axicon microrelief depth is $h=\lambda / 2(n-1)=\lambda$ at $n=1.5$ (refractive index), whereas the central axicon part can be considered as a fragment of a circular fiber with the core radius of $r_{0}=T / 2$.

The number of modes in the circular stepped-index fiber is derived from the dispersion equation [15]:

$$
\frac{u J_{0}(u)}{J_{1}(u)}=-\frac{w I_{0}(w)}{I_{1}(w)},
$$

where $J_{0}, J_{1}, I_{0}, I_{1}$ are the standard and modified Bessel functions of zero and first orders, $u^{2}+w^{2}=V^{2}, V=$ $k r_{0}\left(n_{1}^{2}-n_{2}^{2}\right)^{1 / 2}$, where $n_{1}$ and $n_{2}$ are the refractive indices of the fiber core and cladding, and $r_{0}$ is the core radius.

Because the maximal root of (16) for a waveguide mode is less than the cutoff number, $u_{\max }<V$, we may infer 
that the waveguide mode with a minimal diameter has the amplitude proportional to the Bessel function: $J_{0}\left(V_{r} / r_{0}\right), r<$ $r_{0}$. Hence, the diameter of a mode with the maximal number is $(n=1.5)$

$$
2 r=2 \frac{2.4 r_{0}}{V}=\frac{2.4 \lambda}{\pi \sqrt{n^{2}-1}} \approx 0.7 \lambda .
$$

It is noteworthy that in a similar way to the estimate (11), the diameter estimate (17) is also independent of the axicon period $T$.

2.3. Nonparaxial Relations for the Longitudinal Intensity. The relation between the central peak diameter of the light field and the distance to the axicon should be similar to that between the axial intensity and the distance to the axicon. Actually, if there is a bright ring on the axis, its diameter is supposed to be larger than the diameter at focus, and, on the opposite, the axial intensity has a local maximum at focus and a minimum in the ring center. Thus, we will arrive at the relation for the axial intensity of light produced by the binary axicon. The axial amplitude of the scalar nonparaxial field from a circular aperture of radius $R$ as a function of the longitudinal coordinate is given by [16]

$$
E_{y}(z)=e^{i k z}-\frac{z e^{i k \sqrt{R^{2}+z^{2}}}}{\sqrt{R^{2}+z^{2}}} .
$$

For a binary axicon of transmittance in (1), we can derive a similar relation for the electric field amplitude on the optical axis:

$$
\begin{aligned}
E_{y}(z)= & e^{i \phi}\left[e^{i k z}-\frac{z e^{i k \sqrt{r_{2 N+1}^{2}+z^{2}}}}{\sqrt{r_{2 N+1}^{2}+z^{2}}}\right] \\
& +\left(e^{i \phi}-1\right) z \sum_{m=1}^{2 N} \frac{e^{i k \sqrt{r_{m}^{2}+z^{2}}}}{\sqrt{r_{m}^{2}+z^{2}}}(-1)^{m},
\end{aligned}
$$

where $r_{m}=m r_{0}$ is the radius of the axicon binary relief step and $r_{0}=T / 2$ is half the axicon period.

From (19) it follows that at $\varphi=\pi$, secondary spherical waves scattered from any point of relief jump (i.e., with halfperiod intervals) will be contributing to any point on the $z$ axis. Because the number of the said points equals $2 N+1$, it would be difficult to predict the result of such multiray interference. It can only be said that for $z \ll R$, (19) reduces to (18), suggesting that in the vicinity of the axicon surface the minimal period of the axial intensity variations is as small as $\lambda$.

\section{Fabrication of Binary Axicons}

Three diffractive binary axicons of periods $4 \mu \mathrm{m}, 6 \mu \mathrm{m}$, and $8 \mu \mathrm{m}$ and diameter $4 \mathrm{~mm}$ were fabricated. The process involved the deposition of a $100 \mathrm{~nm}$ thick chromium layer onto a silica substrate $(n=1.46)$ of thickness $1 \mathrm{~mm}$ and diameter $30 \mathrm{~mm}$ using the unit UVM2M1.

On this chromium-plated substrate was written a photomask on a circular laser writing system CLWS-200 of minimal laser spot diameter $0.8 \mu \mathrm{m}$ and a $20 \mathrm{~nm}$ positioning accuracy. In the process of the photomask writing, a protective oxide film was produced on the chromium surface through its exposure to a focused light of the Ar laser of wavelength $500 \mathrm{~nm}$. Then the unoxidized chrome was removed in a solution of potassium bichromate and alkali. In the next step, the photomask underwent plasmochemical etching. The etching was conducted on the unit UTP PDE125-009, producing the relief features of horizontal measures less than $100 \mathrm{~nm}$. The silica was etched at the following parameters: power of high-frequency current, $800 \mathrm{~W}$, interim vacuum $2.1 * 10^{-2} \mathrm{~Pa}$, and current of the plasma-trapping electromagnet, $0.8 \mathrm{~A}$. The etching was conducted in the Freon-12 atmosphere for 21 minutes. The etch rate of the silica substrate was $20-25 \mathrm{~nm}$ per minute. Using the abovedescribed procedure, three axicons $4 \mathrm{~mm}$ in diameter and with periods $4 \mu \mathrm{m}, 6 \mu \mathrm{m}$, and $8 \mu \mathrm{m}$ were fabricated.

Figure 1(a) depicts the SEM image (top view) of the binary axicon-on-silica of period $4 \mu \mathrm{m}$. The image was produced on the scanning electron microscope SUPRA-25 with 1000x magnification. Figure 1(b) shows the image (top view) of the binary axicon with period $8 \mu \mathrm{m}$ obtained with an atomic force microscope Solver Pro.

Figures $1(\mathrm{c})$ and $1(\mathrm{~d})$ show oblique images of the binary axicon reliefs obtained on the Solver Pro microscope: (a) a peripheral part of the axicon with period $6 \mu \mathrm{m}$ and (b) the central part of the axicon with period $8 \mu \mathrm{m}$.

Shown in Figure 2 is the radial section of the peripheral part of relief of the binary axicon of period $6 \mu \mathrm{m}$ (Figure 1(c)), which suggests that the relief depth is $450-500 \mathrm{~nm}$. Because all three axicons in question are designed for the wavelength $532 \mathrm{~nm}$, so the required relief depth is

$$
h=\frac{\lambda}{2(n-1)} \approx 578 \mathrm{~nm} .
$$

Thus, the etch depth error is $20 \%$. It is clearly seen from Figure 4 that the relief top features have a slope of about $1 / 150 \mathrm{rad}$, whereas the bottom has irregularities of height $30 \mathrm{~nm}$. Note, also, that the binary relief has a trapezoid shape, the top-to-bottom ratio is $3: 4$. Besides, it is clearly seen from Figure 1(b) that the step width is larger than the step span, with the width-to-span ratio being $2: 1$.

\section{Experimental Results}

The experiment aimed to look into the relation between the central spot diameter and the distance on the optical axis. The binary axicons were sequentially placed into an optical setup shown in Figure 3, where the near-field diffraction pattern was measured with a CCD-camera at different distances upon illumination by a laser light of wavelength $532 \mathrm{~nm}$.

The beam of a solid-state laser of wavelength $\lambda=532 \mathrm{~nm}$ and diameter $1.4 \mathrm{~mm}$ was focused with a microlens $L_{1}$ into a point diaphragm of diameter $15 \mu \mathrm{m}$. The central part of a uniform light spot produced by the diaphragm was collimated with a lens $L_{2}$, producing a light beam in the form of a bounded near-plane wave. The central portion of 


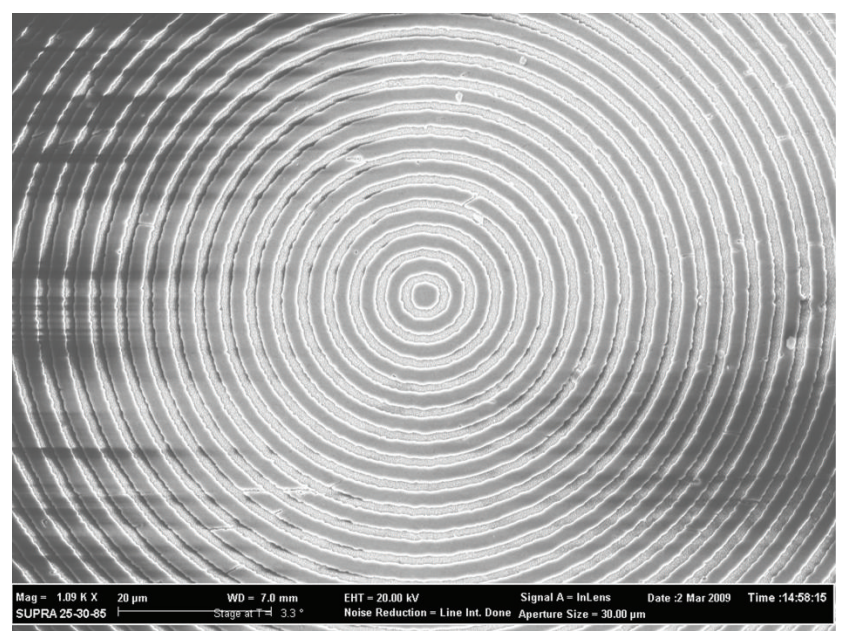

(a)

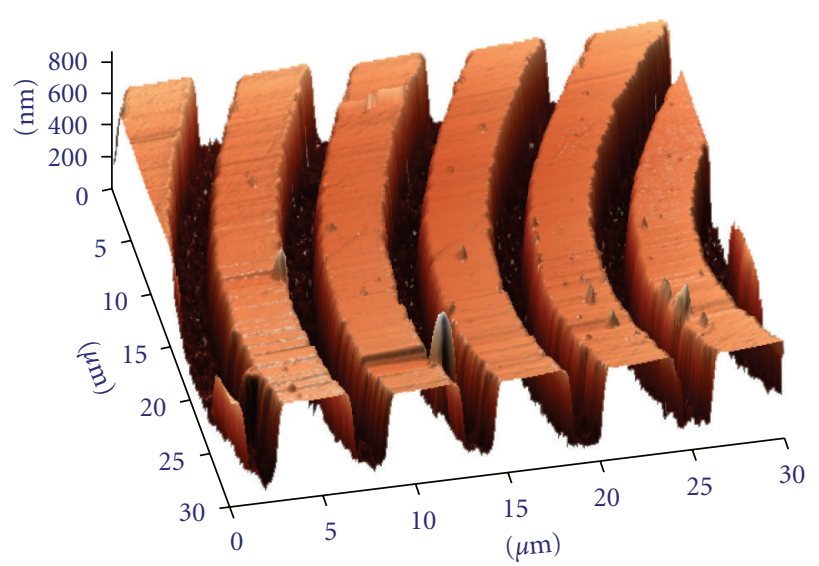

(c)

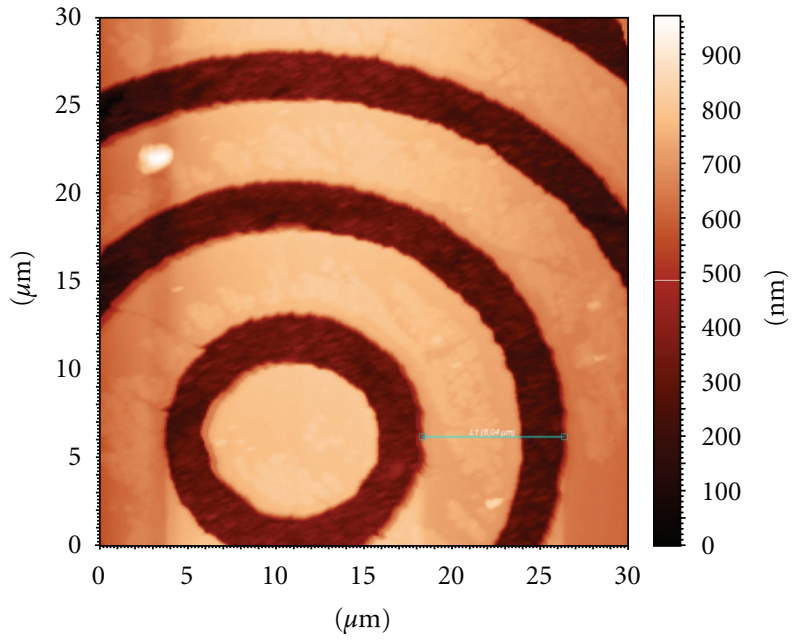

(b)

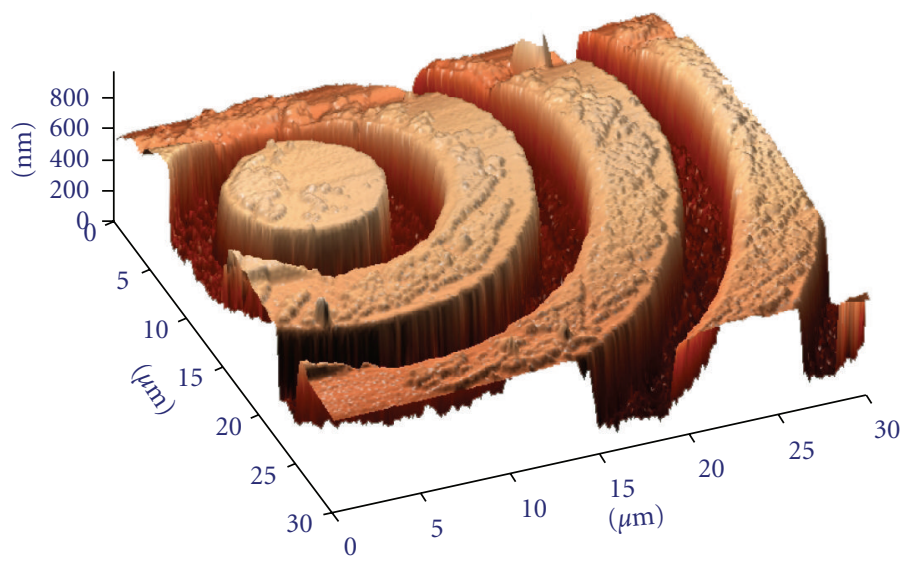

(d)

FIgURE 1: (a) SEM image (top view) of the binary axicon of period $4 \mu \mathrm{m}$, produced with a scanning electron microscope Supra-25 with 1000x magnification. (b) Top view of the binary axicon with period $8 \mu \mathrm{m}$, produced with an atomic force microscope Solver Pro. An oblique image of (c) the peripheral fragment of the binary axicon with period $6 \mu \mathrm{m}$ and (d) the central part of the binary axicon of period $8 \mu \mathrm{m}$, produced with the Solver Pro microscope.

the resulting beam was coupled into an optical system of the microscope Biolam-M and then focused by a microlens $L_{3}$, so that the laser beam diameter should be equal to the axicon diameter $D_{3}$ (the matching of the beam and axicon diameters was intended to reduce the energy losses). The resulting diffraction pattern was recorded on a CCD-camera with the aid of a microlens $L_{4}$. By displacing the axicon $D_{3}$, we can obtain the diffraction patterns at different distances from the element. The origin of coordinates was placed on the microrelief valley surface, which was brought to sharp focus in white light. The axicon $D_{3}$ was displaced with a micrometric screw with a $1-\mu \mathrm{m}$ division value. The CCDcamera resolution was $2048 * 1536$ pixels, with the pixel size being $6.9 \mu \mathrm{m}$.

Figure 4 shows the central maximum diameter as a function of distance from the axicon surface (with respect to a transverse Cartesian coordinate) for period (a) $4 \mu \mathrm{m}$ and (b) $8 \mu \mathrm{m}$.
It is seen from Figure 4 that in the interval from 0 to $40 \mu \mathrm{m}$, the central maximum diameter is changing quasiperiodically with a period of about $2 \mu \mathrm{m}$ (Figure $4(\mathrm{a})$ ) and $4 \mu \mathrm{m}$ (Figure $4(\mathrm{~b})$ ). Note that the diameter values larger than $5 \lambda$ (Figure 4(a)) and $9 \lambda$ (Figure 4(b)) correspond to the annular intensity distribution centered in the axis (on which it has a local minimum), while the diameter values smaller than $4.5 \lambda$ (Figure $4(\mathrm{a})$ ) and $8 \lambda$ (Figure 4(b)) correspond to the local maxima intensity (focal points). The analysis of Figure 4 suggests that the diameter of the near-field axial focal spots (at the distance $\leq 40 \mu \mathrm{m}$ ) is varied from $3.5 \lambda$ to $4.5 \lambda$ (Figure $4(\mathrm{a})$ ) with a $0.4 \lambda$ error and from $5 \lambda$ to $8 \lambda$ (Figure $4(\mathrm{~b})$ ) with a $0.5 \lambda$ error, respectively, for the axicons with period $4 \mu \mathrm{m}$ and $8 \mu \mathrm{m}$.

Figure 5 shows the recorded diffraction patterns of laser light produced by binary axicons with period (a), (b) $4 \mu \mathrm{m}$ and (c, d) $8 \mu \mathrm{m}$ at distances of (a) $5 \mu \mathrm{m}$, (b) $8 \mu \mathrm{m}$, (c) $16 \mu \mathrm{m}$, and (d) $18 \mu \mathrm{m}$. From Figure 5, the annular intensity 


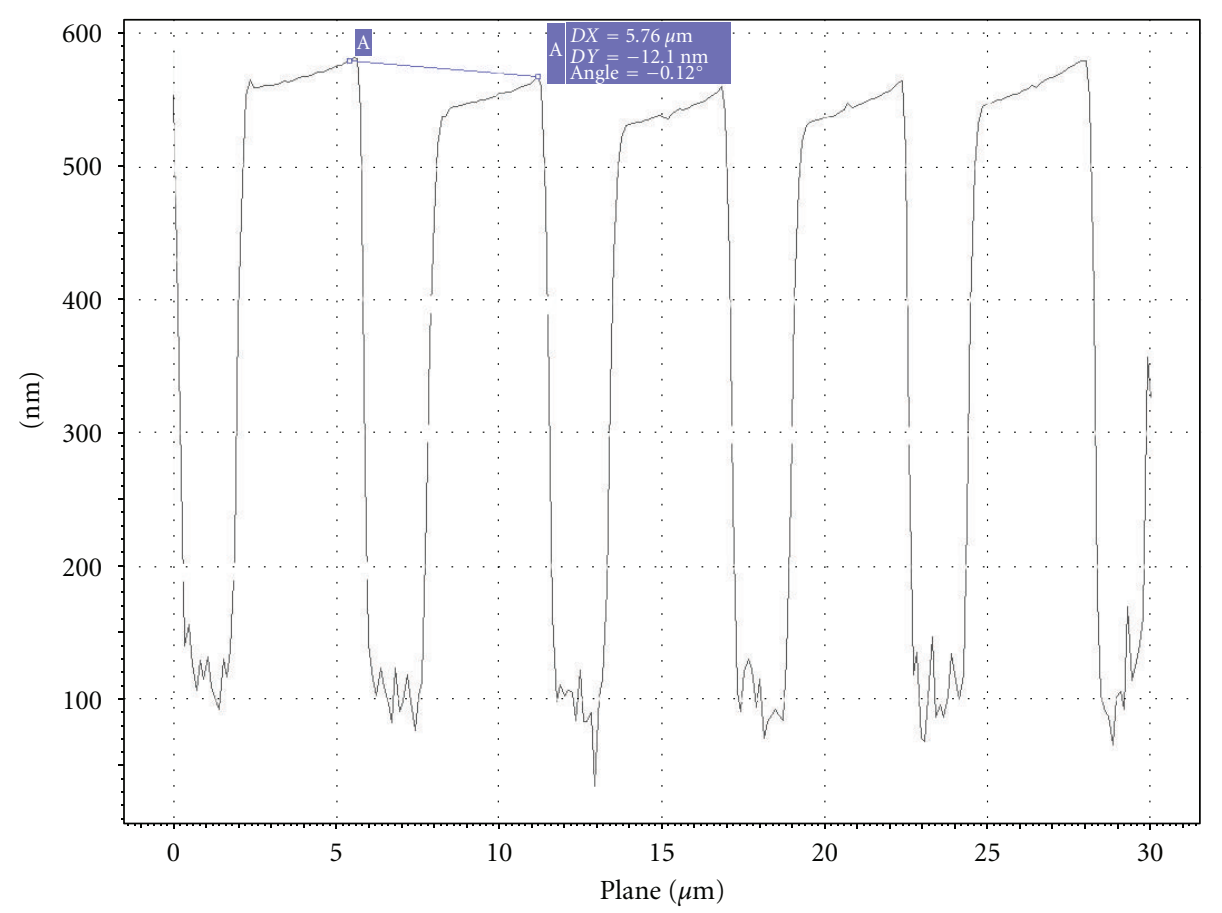

FIGURE 2: Profile of the binary axicon with period $6 \mu \mathrm{m}$ of Figure 1(c), produced on the Solver Pro microscope.

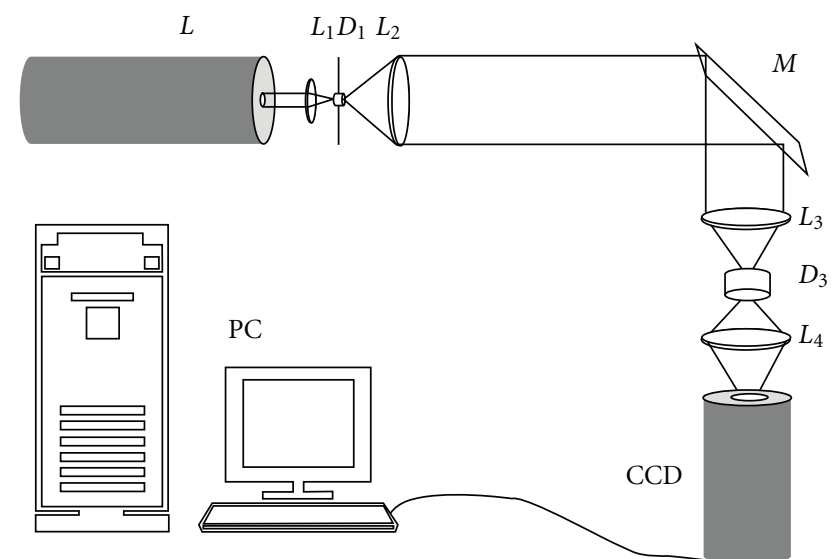

FIGURE 3: An optical arrangement for measuring the near-field diffraction pattern from the binary axicons: $L$ : laser, $L_{1}$ : microlens $(20 \mathrm{x}, \mathrm{NA}=0.4), D_{1}$ : point diaphragm $($ diameter $=15 \mu \mathrm{m}), L_{2}$ : collimating lens $(f=100 \mathrm{~mm}), M$-deflecting mirror, $L_{3}$ : focusing microlens $(8 \mathrm{x}, \mathrm{NA}=0.2), L_{4}$ : imaging microlens $(20 \mathrm{x}, N A=0.4)$, $D_{3}$ : binary axicon.

distribution on the optical axis (a), (c) is seen to be alternated with a central focal spot (b), (d) at 2-3 $\mu$ m intervals over a distance less than $40 \mu \mathrm{m}$. Because the diffraction patterns in Figure 5 are shown in the same scale $(75 \times 55 \mu \mathrm{m})$, it can be seen that the corresponding diameters of the ring and the focus for the axicon of period $8 \mu \mathrm{m}$ are 1.5 times larger than those for the axicon of period $4 \mu \mathrm{m}$.

Figure 6 shows (a) the diffraction pattern from the axicon of period $4 \mu \mathrm{m}$ recorded $2 \mu \mathrm{m}$ away from its surface and the pattern cross-sections on the (b) $x$ - and (c) $y$-axes. It is seen from Figure 6 that at small distances of $z<5 \mu \mathrm{m}$ the focal spot in the diffraction pattern features an ellipticity of eccentricity $\varepsilon=0.63$ (which is not found in Figure 5) associated with the linear polarization of the incident laser light (the electric field of the incident beam directed along the $y$-axis in Figure 6).

\section{Simulation Results}

The diffraction of the linearly polarized plane wave by the diffractive binary axicons was simulated using two similar but still different techniques: the BOR-FDTD method that realizes a finite-difference algorithm for solving Maxwell's equations in cylindrical coordinates using Matlab [17] and the 3D-FDTD method implemented in the FullWAVE (RSoft) program.

Figure 7 depicts the simulated distributions of the squared electric vector magnitude in the $X Z$-plane of a linearly polarized plane wave having passed the binary axicon of period $4 \mu \mathrm{m}$, calculated using two approaches. Figure 7 suggests that both diffraction patterns show a qualitative similarity, which is evidenced by the fact that in both pictures each asperity of the axicon relief focuses light into local foci at a distance of $2 \mu \mathrm{m}$ (Figure 7(a)) and $1.7 \mu \mathrm{m}$ (Figure 7(b)) from the axicon surface. These values agree well with the estimate that follows from (18). In the paraxial case, it follows from (18) that the intensity along the optical axis will vary according to $\cos \left(k R^{2} / 4 z\right)$ with a period of $z_{0}=$ $R^{2} / 4 \lambda=1.9 \mu \mathrm{m}$ when $T=4 \mu \mathrm{m}$. It should be noted that FullWAVE does not calculate the intensity distribution. It calculates only the amplitude distribution. So we used our software to calculate intensity using amplitude slices at the 


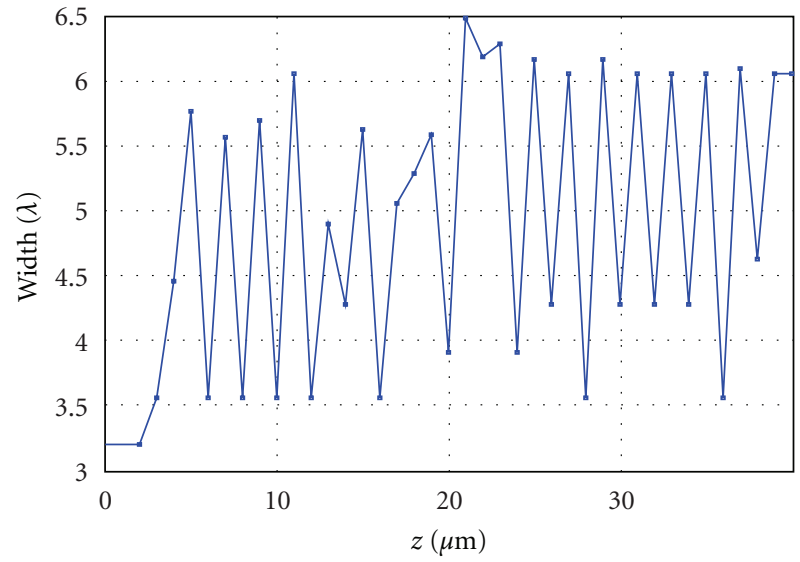

(a)

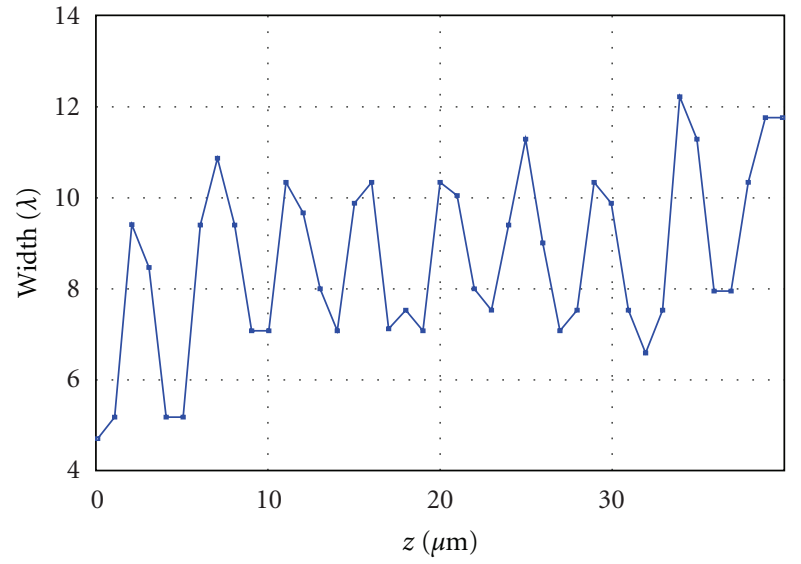

(b)

FIGURE 4: The diameter of the light spot on the axis (in wavelengths) as a function of distance from binary axicons with period (a) $4 \mu \mathrm{m}$ and (b) $8 \mu \mathrm{m}$.

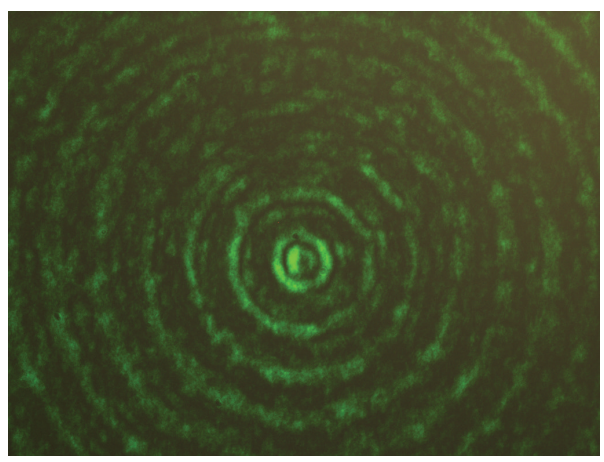

(a)

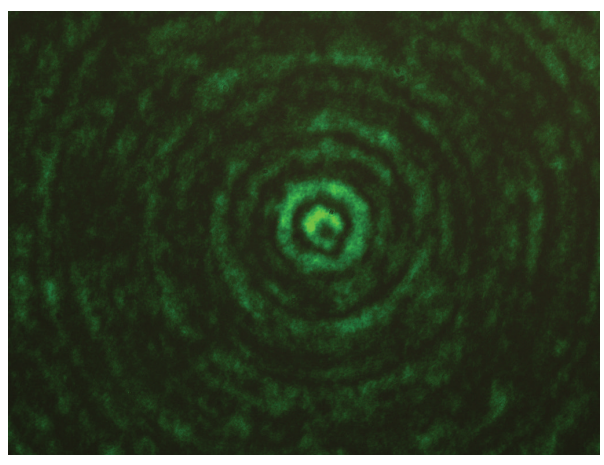

(c)

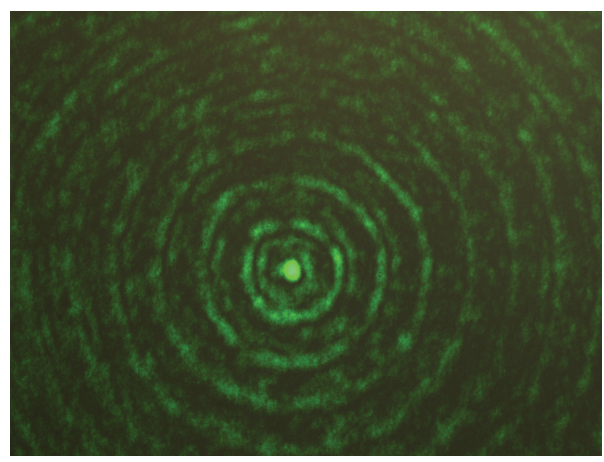

(b)

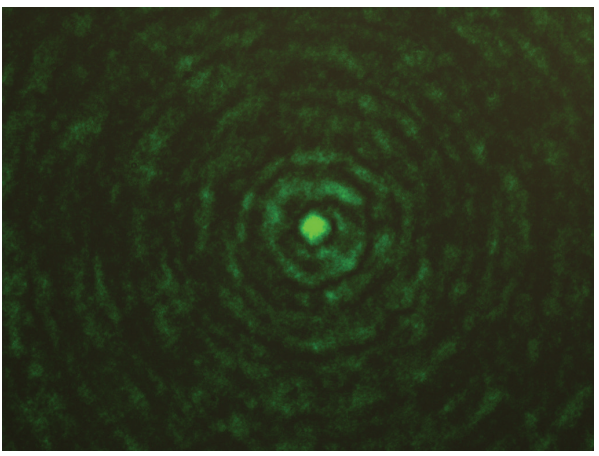

(d)

Figure 5: Diffraction patterns recorded with the CCD-camera from the axicons with period (a), (b) $4 \mu \mathrm{m}$ and (c), (d) $8 \mu \mathrm{m}$ at different distances: (a) $5 \mu \mathrm{m}$, (b) $8 \mu \mathrm{m}$, (c) $16 \mu \mathrm{m}$, and (d) $18 \mu \mathrm{m}$.

different time steps. To calculate intensity distribution in FullWAVE we average a square of amplitude during the all modeling time, but in our BOR-FDTD implementation we average square of amplitude in the last period of modeling. This explains the quantitative difference between the results.

Shown in Figure 8 are (a) the diffraction patterns in the $X Y$-plane at distance $z=1.7 \mu \mathrm{m}$ (in the local focus plane), (b) its radial section, and, for comparison, the intensity distribution in the $X Y$-plane at a distance of $z=0.3 \mu \mathrm{m}$ at which a light ring is formed on the optical axis.

From Figures 8(a) and 8(b), the local maximum diameter can be estimated at $1.25 \lambda(\lambda=532 \mathrm{~nm})$. Comparing this value with the experimentally measured diameter of the local focal spot in Figure 6 (of diameter 3.6 $\lambda$ on the $x$-axis), we can see that the former is nearly three times smaller. 


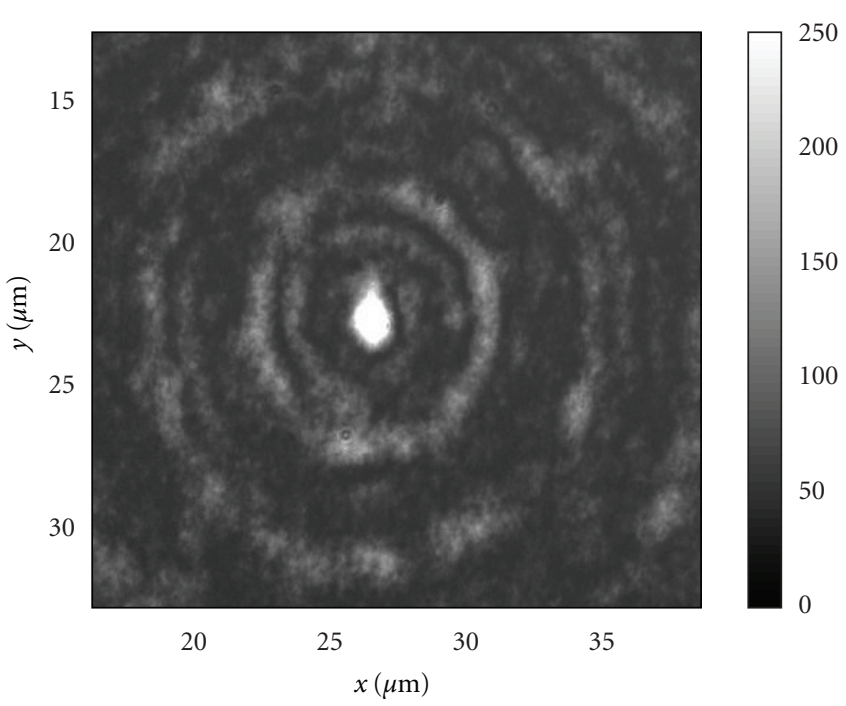

(a)

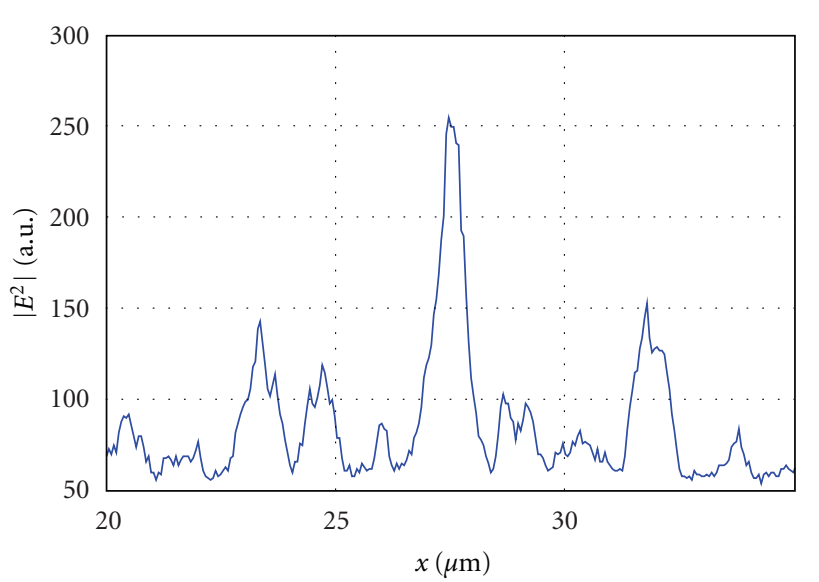

(b)

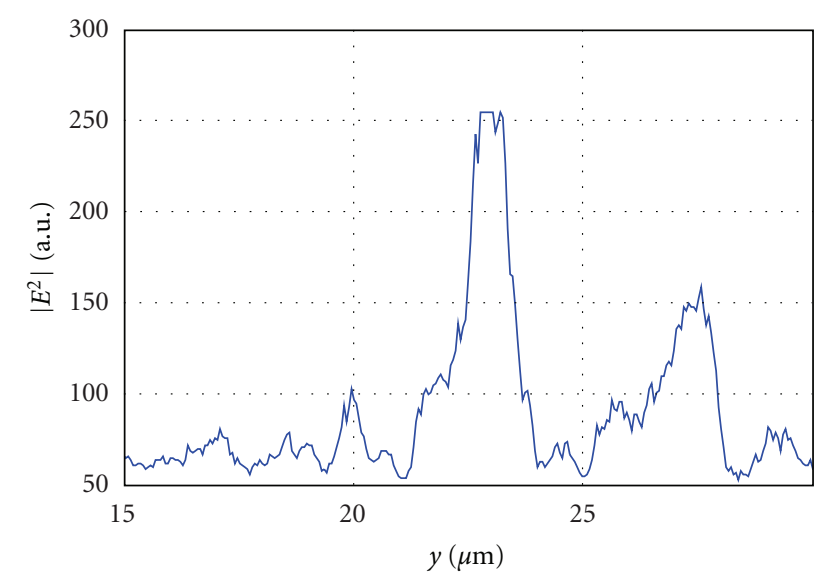

(c)

Figure 6: (a) The intensity distribution recorded at a $2 \mu \mathrm{m}$ distance from the binary axicon of period $4 \mu \mathrm{m}$ and the cross-sections thereof on the (b) $x$-and (c) $y$-axes.

Figure 9 depicts, derived with the BOR-FDTD method, the axial intensity distribution for an axicon of radius $28 \mu \mathrm{m}$ and period $4 \mu$, which is illuminated by a linearly polarized plane wave.

From Figure 9, the local peaks are seen to alternate on the optical axis in a quasiperiodic manner, with the peak-topeak spacing increasing from $2 \mu \mathrm{m}$ to $8 \mu \mathrm{m}$ over the distance $z<60 \mu \mathrm{m}$.

Figure 10 depicts the axial intensity distribution (in arbitrary units) for the binary axicon of period $4 \mu \mathrm{m}$ and radius $28 \mu \mathrm{m}$. As distinct from Figure 9, the distribution of Figure 10 resulted from illuminating the axicon by a linearly polarized diverging Gauss beam, with the electric vector amplitude given by

$$
E_{y}(r, z=0)=\exp \left(-\frac{r^{2}}{w^{2}}+i \frac{k r^{2}}{2 f}\right)
$$

where $w=2 \mathrm{~mm}$ is the waist radius of the Gauss beam, $f=2.5 w$ is the focal length of a parabolic lens. The use of the diverging beam (in contrast with the previously studied plane beam) was prompted by the effort to match the simulation with the experimental results arrived at using the setup in Figure 3. In the arrangement of Figure 3, the binary axicon $D_{3}$ of diameter $4 \mathrm{~mm}$ was illuminated by a diverging laser light having passed the microlens $L_{3}$. The diverging light beam was used to match the diameter of the illuminating light spot with that of the axicon, $D_{3}$. In Figure 10, the peak-to-peak spacing is increasing from $2 \mu \mathrm{m}$ to $4 \mu \mathrm{m}$ at $z<50 \mu \mathrm{m}$. The comparison of the plots in Figures 9 and 10 shows that in the interval $0<z<30 \mu \mathrm{m}$ the periods of intensity oscillations are nearly the same, while in the interval $30<z<50 \mu \mathrm{m}$ the intensity oscillation period in Figure 10 is smaller.

Figure 11 depicts, calculated with the BOR-FDTD method, diameters of the central spots produced by the diverging wave of (21) as a result of diffraction from the axicon of period $T=4 \mu \mathrm{m}$ versus the distance to the axicon. 


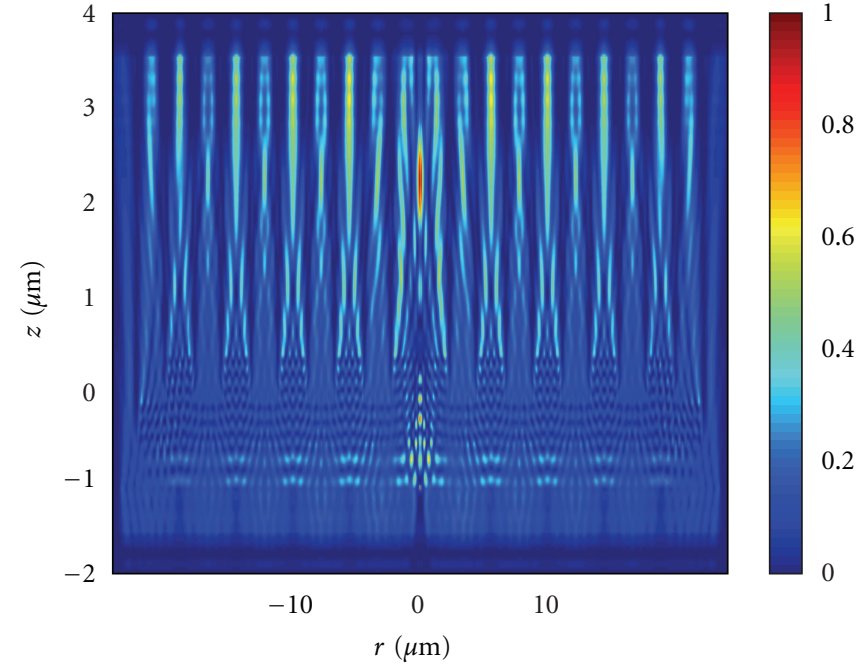

(a)

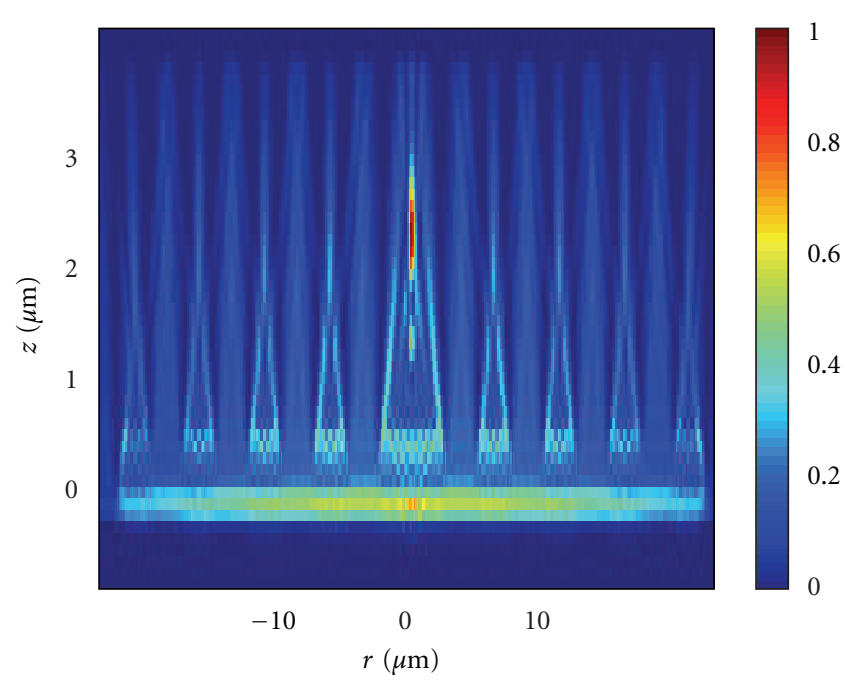

(b)

Figure 7: Simulated intensity distribution in the $X Z$-plane when a linearly polarized plane wave (the electric vector directed along the $Y$-axis) is diffracted by a binary axicon of period $4 \mu \mathrm{m}$ : (a) Matlab2008a, (b) FullWAVE (RSoft).

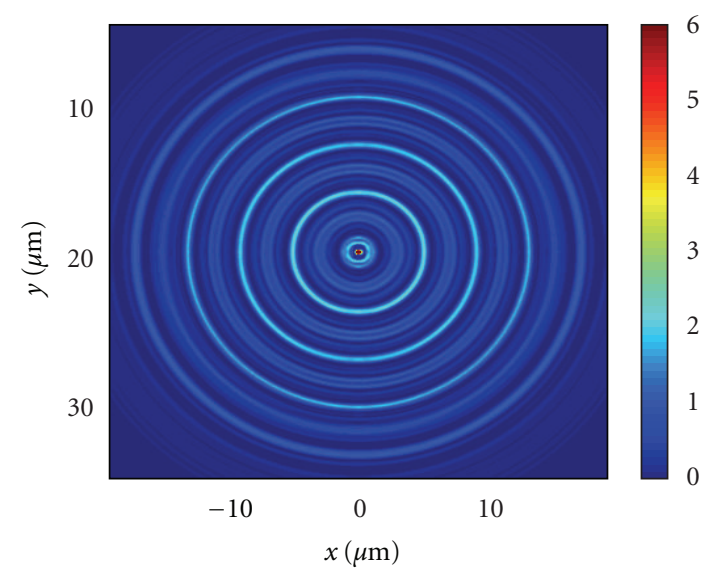

(a)

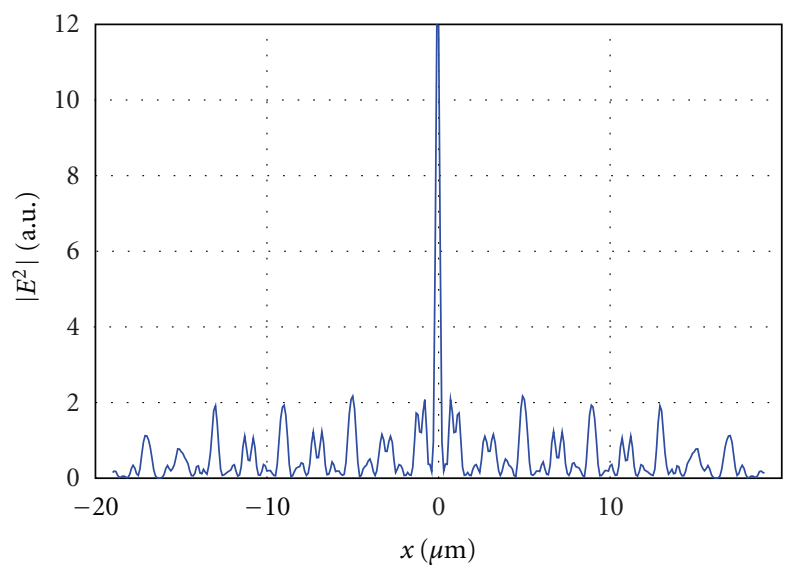

(b)

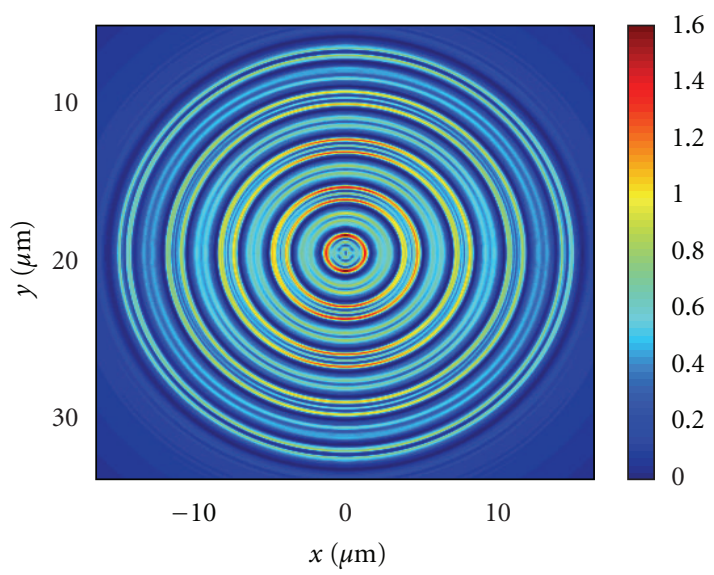

(c)

FIGURE 8: The intensity distribution in the transverse $X Y$-plane at distance (a) $z=1.7 \mu \mathrm{m}$, (c) $z=0.3 \mu \mathrm{m}$, and (b) the radial section of the distribution in (a) for the binary axicon of period $T=4 \mu \mathrm{m}$, simulated using FullWAVE. 


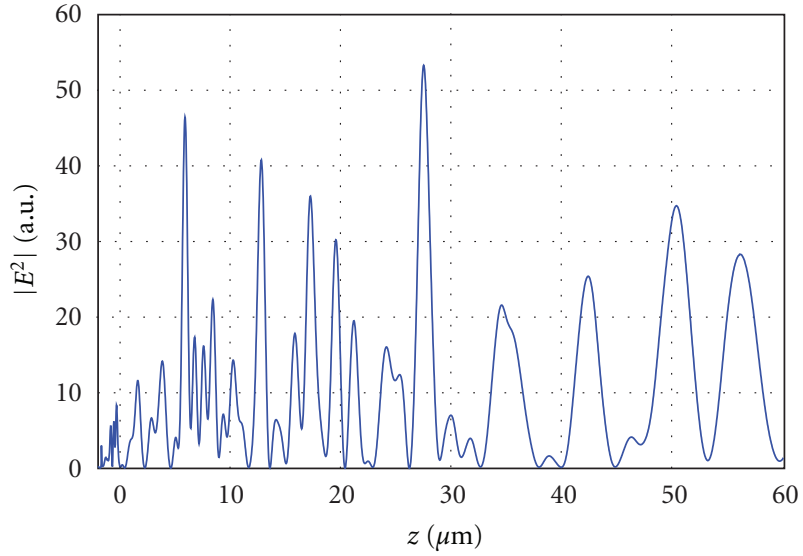

Figure 9: The axial intensity distribution for the axicon of period $4 \mu \mathrm{m}$ illuminated by a linearly polarized plane wave, simulated with the BOR-FDTD method.

Figure 11 shows local maxima that correspond to the annular intensity distribution in the central spot of the diffraction pattern, whereas local minima correspond to the focal spots on the optical axis. From Figure 11, the diameter of the focal spots on the optical axis produced by the binary axicon of period $4 \mu \mathrm{m}$ is also seen to vary in the near field $(z<40 \mu \mathrm{m})$ from $2 \lambda$ to $4.3 \lambda$ with a longitudinal period of about $3 \mu \mathrm{m}$ (with 12 local minima found at length $40 \mu \mathrm{m}$ in Figure 11). From the comparison of the experimental and simulated curves for the central spot diameters in the diffraction pattern (Figure 4(a) and Figure 11) it follows that the focal spot diameters conform well at a distance up to $40 \mu \mathrm{m}$ : from $3.5 \lambda$ to $4.5 \lambda$ (Figure $4(\mathrm{a})$ ) and from $2 \lambda$ to $4.3 \lambda$ (Figure 11). Note, however, that the longitudinal period of the focal spot variation was $3 \mu \mathrm{m}$ in Figure 11, whereas in the experiment it was $2 \mu \mathrm{m}$.

Comparison of Figures 10 and 11 shows that there is agreement in the number of the local foci (intensity minima in Figure 11 and maxima in Figure 10): in the interval $30 \mu \mathrm{m}<z<40 \mu \mathrm{m}$, there are 4 minima in Figure 11 and 4 maxima in Figure 10; meanwhile in the interval $20 \mu \mathrm{m}$ $<z<30 \mu \mathrm{m}$ there are 2 minima in Figure 11 and two maxima in Figure 10.

From (15) it follows that the focal spot diameter produced by the axicon of period $4 \mu \mathrm{m}$ eqauls $2 r=0.774 T=$ $3.1 \mu \mathrm{m}=6 \lambda$ (for $m=1$ ) and $3 \lambda$ (for $m=2$ ). Comparing the above values with the experimental (Figure 4(a)) and simulation data (Figure 11) we can infer that for the nearfield diffraction the contribution to the foci on the optical axis comes from the first and second diffraction orders of the binary axicon.

\section{Conclusion}

We have obtained the following results.

(i) It has been shown that the calculation of the nonparaxial complex amplitude of the light field resulting from the diffraction of a plane wave by a binary axicon reduces to calculating the integral of a product

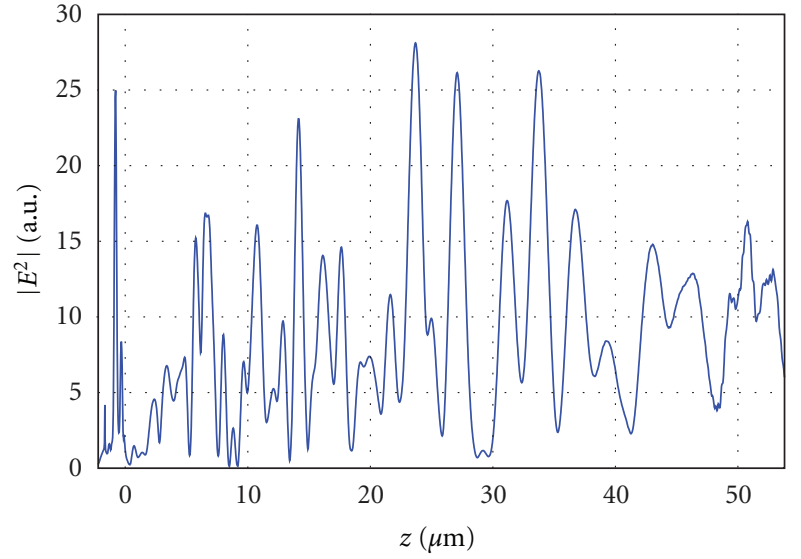

FIGURE 10: The axial intensity distribution calculated using the BOR-FDTD method for the axicon of period $4 \mu \mathrm{m}$ and radius $28 \mu \mathrm{m}$ illuminated by a linearly polarized diverging Gauss beam.

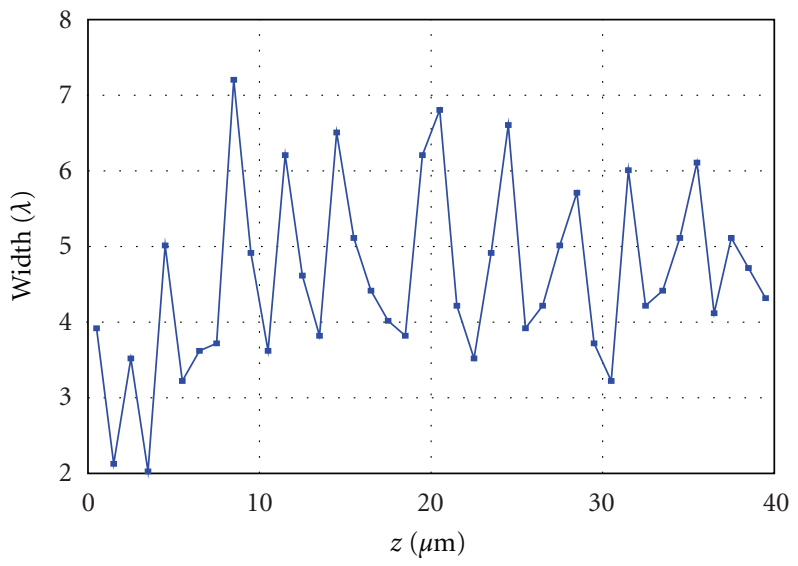

FIGURE 11: BOR-FDTD simulation: the profile of the full-width (diameter on the $x$-axis in wavelengths) of the central spot intensity of the diverging laser beam diffracted by the binary axicon of period $4 \mu \mathrm{m}$.

of two Bessel functions of the zero and first orders (3), (4).

(ii) By approximate calculations, we have shown that at $z \ll \lambda$, the central maximum diameter of the light field is independent of the axicon period, being equal to the diffraction limit of $0.6 \lambda$ (11). The said estimate of the diameter of the nearaxicon focal spot conforms with the estimate of the minimal modulation diameter $(0.7 \lambda)$ of the light field composed of spatial modes of a stepped-index fiber whose diameter equals the axicon period (17).

(iii) A relationship has been deduced of the field complex amplitude on the optical axis for a plane wave diffracted from a binary axicon (19), which suggests that in the axicon vicinity, the minimal period of axial intensity oscillations is $\lambda$.

(iv) Using a laser writing system CLWS-200 and plasmo-chemical etching of a fused-silica substrate 
( $n=1.46)$, we fabricated binary axicons of periods $4 \mu \mathrm{m}, 6 \mu \mathrm{m}$, and $8 \mu \mathrm{m}$, diameter $4 \mathrm{~mm}$, and depth $500 \mathrm{~nm}$. The analysis of the axicon surface with a Solver Pro microscope has shown that the axicon was fabricated with a $20 \%$ error. Top features of the relief have a slope of about $0.01 \mathrm{rad}$, the binary relief steps are a 3:4 trapezoid, and the relief bottom has $30 \mathrm{~nm}$ asperities.

(v) Experimental relationships for the variation of the focal spot diameter along the optical axis in the near field of the binary axicon $(z<40 \mu \mathrm{m})$ have been derived, showing that for the axicon of period $4 \mu \mathrm{m}$, the focal spot diameter varies from $3.5 \lambda$ to $4.5 \lambda$ with a $2 \mu \mathrm{m}$ period, and for the axicon of period $8 \mu \mathrm{m}-$ from $5 \lambda$ to $8 \lambda$ with a $4 \mu \mathrm{m}$ period (Figure 4 ). This result agrees with [13].

(vi) The minimal period of an elliptic focal spot equal to $3.6 \lambda \mu \mathrm{m}(\mathrm{FWHM}=1.2 \lambda)$ was found at a $2 \mu \mathrm{m}$ distance from the axicon of period $4 \mu \mathrm{m}$ (Figure 6). This result agrees well with the findings reported in $[5,9]$.

(vii) We have experimentally shown that in the nearaxicon region $(z<5 \mu \mathrm{m})$, elliptical focal spots are formed (with eccentricity of 0.63 ), which are elongated along the linear polarization vector of the incident light wave.

(viii) For comparison, the diffraction of a linearly polarized plane wave by the binary axicon of period $4 \mu \mathrm{m}$ and diameter $40 \mu \mathrm{m}$ was simulated using (1) the FullWAVE (by RSoft), which solves Maxwell's equation in the Cartesian coordinates using a 3D-FDTD method and (2) the earlier developed proprietary BOR-FDTD software for solving Maxwell's equations in the cylindrical coordinates by a finite-difference scheme for radially symmetric microoptics elements. In both simulations, the diffraction patterns show that the first focus appears $1.7-2 \mu \mathrm{m}$ apart from the axicon surface (Figure 7).

(ix) The numerically simulated relationship for the central spot diameter variation on the optical axis when a linearly polarized plane wave is diffracted from the binary axicon of period $4 \mu \mathrm{m}$ has been shown to conform with the similar experimental curve (Figures 4(a) and 11): in the interval $0<z<40 \mu \mathrm{m}$, the focal spot diameter on the optical axis varies from $3.5 \lambda$ to $4.5 \lambda$ (experiment) and from $2 \lambda$ to $4.3 \lambda$ (simulation), with the local foci appearing on the axis with period $2 \mu \mathrm{m}$ (experiment) and $3 \mu \mathrm{m}$ (simulation).

\section{Acknowledgments}

The work was financially supported by the Federal Program Research and Educational Staff of Innovation Russia (State Contract no.14.740.11.0016), Russian Federation Presidential Grants for Support of Leading Scientific Schools
(NSh-4128.2012.9) and a Young Researcher's Grant (MK3912.2012.2), the Russian Foundation for Basic Research Grant (12-07-00269).

\section{References}

[1] J. H. McLeod, "The Axicon: a new type of element," Journal of the Optical Society of America, vol. 44, pp. 592-597, 1954.

[2] J. Durnin, "Exact solutions for nondiffracting beams. I. The scalar theory," Journal of the Optical Society of America A, vol. 4, pp. 651-654, 1987.

[3] J. Durnin, J. Miceli, and J. H. Eberly, "Diffraction-free beams," Physical Review Letters, vol. 58, no. 15, pp. 1499-1501, 1987.

[4] J. K. Kim, J. Kim, Y. Jung et al., "Compact all-fiber Bessel beam generator based on hollow optical fiber combined with a hybrid polymer fiber lens," Optics Letters, vol. 34, no. 19, pp. 2973-2975, 2009.

[5] H. Kurt, "Limited-diffraction light propagation with axiconshape photonic crystals," Journal of the Optical Society of America B: Optical Physics, vol. 26, no. 5, pp. 981-986, 2009.

[6] W. Chen and Q. Zhan, "Realization of an evanescent Bessel beam via surface plasmon interference excited by a radially polarized beam," Optics Letters, vol. 34, no. 6, pp. 722-724, 2009.

[7] K. Watanabe, G. Terakado, and H. Kano, "Localized surface plasmon microscope with an illumination system employing a radially polarized zeroth-order Bessel beam," Optics Letters, vol. 34, no. 8, pp. 1180-1182, 2009.

[8] Y. Fu and W. Zhou, "Hybrid Au-Ag subwavelength metallic structures with variant periods for superfocusing," Journal of Nanophotonics, vol. 3, Article ID 033504, 2009.

[9] Y. Fu, R. G. Mote, Q. Wang, and W. Zhou, "Experimental study of plasmonic structures with variant periods for subwavelength focusing: analyses of characterization errors," Journal of Modern Optics, vol. 56, no. 14, pp. 1550-1556, 2009.

[10] P.-K. Wei, W.-L. Chang, K.-L. Lee, and E.-H. Lin, "Focusing subwavelength light by using nanoholes in a transparent thin film," Optics Letters, vol. 34, no. 12, pp. 1867-1869, 2009.

[11] E. Schonbrun, W. N. Ye, and K. B. Crozier, "Scanning microscopy using a short-focal-length Fresnel zone plate," Optics Letters, vol. 34, no. 14, pp. 2228-2230, 2009.

[12] P. Vahimaa, V. Kettunen, M. Kuittinen, J. Turunen, and A. T. Friberg, "Electromagnetic analysis of nonparaxial Bessel beams generated by diffractive axicons," Journal of the Optical Society of America A, vol. 14, no. 8, pp. 1817-1824, 1997.

[13] Y. Kizuka, M. Yamauchi, and Y. Matsuoka, "Characteristics of a laser beam spot focused by a binary diffractive axicon," Optical Engineering, vol. 47, Article ID 053401, 2008.

[14] A. P. Prudnikov, Y. A. Brychkov, and O. I. Marichev, Integrals and Series. Special Functions, Nauka, Moscow, Russia, 1983.

[15] A. Snyder and J. Love, Optical Waveguide Theory, Kluwer Academic/Plenum, New York, NY, USA, 2000.

[16] H. Osterberg and L.W. Smith, "Closed solutions of Rayleigh's diffraction integral for axial points," Journal of the Optical Society of America, vol. 51, pp. 1050-1054, 1961.

[17] V. V. Kotlyar and S. S. Stafeev, "Sharply focusing a radially polarized laser beam using a gradient Mikaelian's microlens," Optics Communications, vol. 282, no. 4, pp. 459-464, 2009. 

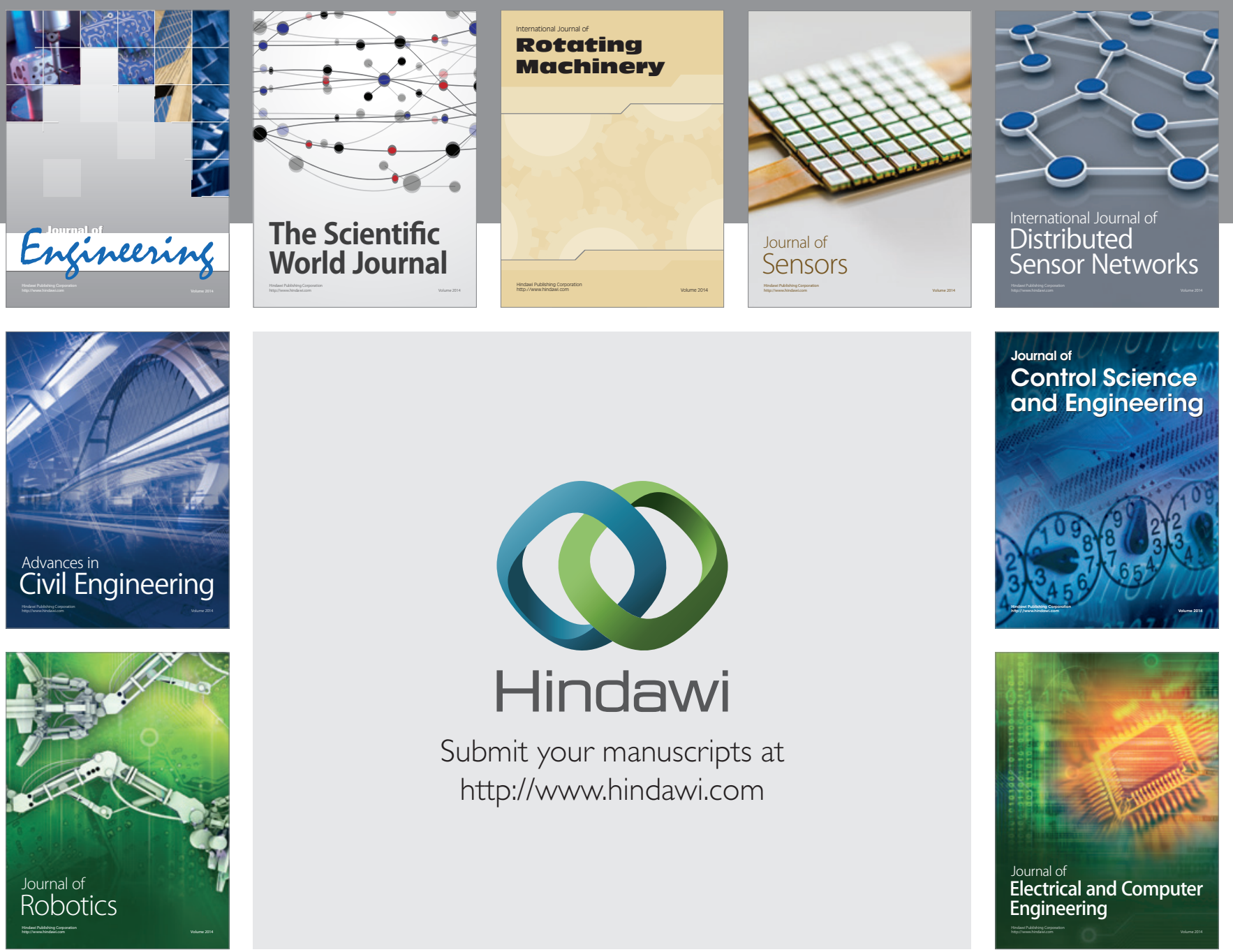

Submit your manuscripts at

http://www.hindawi.com
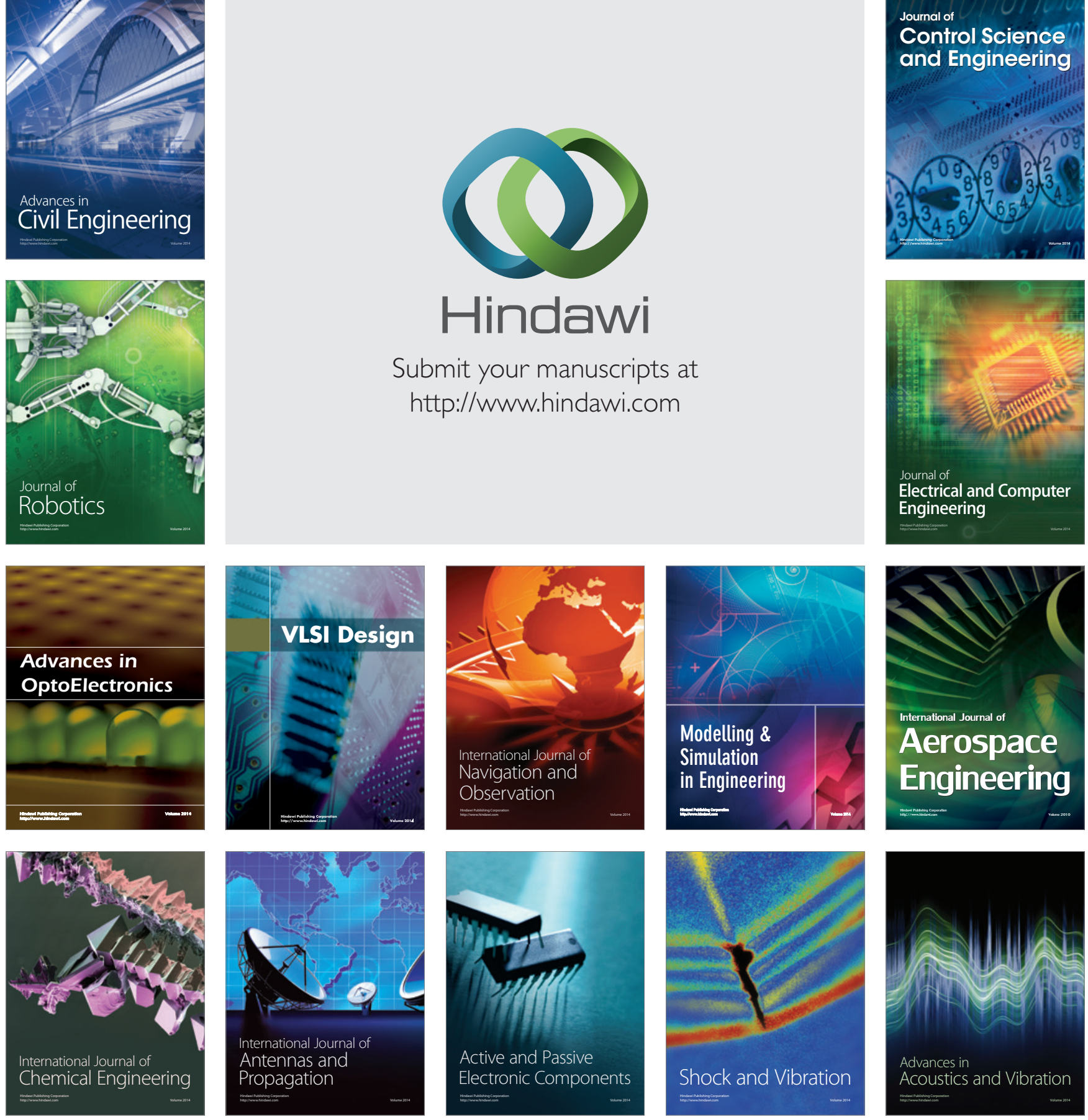